there may be still enough vitamin about to run essential biochemical processes. If, however, there is also the ill-health suggested, it is important; but its very vagueness must increase the need for caution and for prolonged experiments (taking into account all psychological factors) before coming to a final judgment. It is dificult to see how the state of the individual (as distinct from statistical averages over a group) can be assessed without combining blood estimations with clinical examinations, as Dr. Sinclair and his colleagues are trying to do. So much for the purely scientific attitude. Meanwhile we are faced with practice in the War. We know from McCance and Widdowson that there is a wide difference in calcium requirements of individuals ; we have the League of Nations standards for the earlier vitamin factors, but none for those more recently discovered ; we know that even animals are individual in their needs of a vitamin like aneurin. Let us hope that ultimately we may be able to recognize on a scientific basis cases where intake of a special factor must be increased for an individual. For the moment we are left with the dilemma that diets must provide sufficient margin of accessory factors to ensure no physical inefficiency, and we must see that time and valuable foodstuffs (synthetic and natural) are not wasted; fortunatsily, the latter does not apply to chalk and iron.

R. A. Peters.

\section{AMINO ACID ANALYSIS AND THE STRUCTURE OF PROTEINS*}

\section{By PROF. A. C. CHIBNALL, F.R.S.}

TN recent years $X$-ray crystallographers have made 1 remarkable advances in the interpretation of protein structure, and it is becoming more and more evident that a stage has been reached when their views need to be reconciled with data obtained from accurate amino-acid analysis of the proteins concerned. In 1934 Astbury suggested that in gelatin the residues of glycine and hydroxyproline must repeat at regular intervals along the peptide chain, an idea developed in the following year by Bergmann and Niemann into a general hypothesis that (briefly) the 'frequency' of recurrence of any particular residue must be factorizable entirely by 2 and/or 3 , as must also the total number of residues in the molecule. Evidence in its favour was adduced from analytical data for several proteins, including eggalbumin. The hypothesis has been criticized on various grounds, chief among them being that existing methods of analysis are in most cases not sufficiently quantitative to warrant the results being interpreted with the precision needed. What are believed to be quantitative estimations for the bases and dicarboxylic acids in edestin, egg-albumin and $\beta$-lactoglobulin have now been obtained.

Edestin.-The analytical data and calculated mean residue weight $(115 \cdot 7)$ show that the smallest possible unit contains $432\left(2^{4} \times 3^{3}\right)$ residues, and that the number of residues of arginine, histidine, lysine, tyrosine, tryptophan, methionine and cystine are $48,8,8,12$, 4,8 and 3 respectively. On the assumption that the amide-nitrogen can be distributed in such a way that the number of residues of asparagine, aspartic acid, glutamine and glutamic acid are also factors of 432 , it can be shown that these must be $27,18,36$

- Substance of the Bakerian Iecture delivered before the Royal Society on July 16 . and 36 respectively. The data lend support to the Bergmann and Niemann hypothesis, but at present there is no evidence that edestin is homogeneous.

$\beta$-Lactoglobulin is homogeneous. The analytical data suggest a molecular weight of 42,000 , in agreement with physical measurement, but the total number of residues is not factorizable by 2 and/or 3 . The Bergmann-Niemann hypothesis is thus not valid. On the assumption that the molecule is one peptide chain, the number of carboxyl groups computed from titration curves is in agreement with the chemical data, but the number of free amino groups is eight more than can be attributed to lysine. The molecule is a system of nine peptide chains, not all of like composition, coupled by linkages involving carboxyl and not amino groups. Imide and thiolester linkages are suggested; the presence of the latter would explain many puzzling features of denaturation.

Egg-albumin is not homogeneous and tells a somewhat similar story. Chemical data suggest a molecular weight of 43,000 , in conformity with physical measurements; the Bergmann-Niemann hypothesis does not hold. From titration and other data it is suggested that the molecule is a system of at least four peptide chains having free terminal amino groups, recalling Astbury's deduction that the denatured protein is a pile of four laminæ.

Insulin.-If the excess of free amino groups is a measure of the number of peptide chains, as in lactoglobulin, the molecule of insulin must be a system of eighteen such chains, a striking confirmation of Bernal's prediction from the peaks in the Patterson projection.

Reverting to edestin, it has been found that the determination of free amino groups is complicated by edestan formation. It is probable that the submolecule of 50,000 is one peptide chain, though the possibility that it is a system of three peptide chains of unlike composition cannot be dismissed, in which case the allocation of the amide-nitrogen given above would not be justified. Nevertheless, the stoichiometric relationships exhibited by the other residues are sufficiently impressive to warrant the assumption that the constituent peptide chains would be built to essentially the same pattern based on $2^{n} \times 3^{m}$, though they might not contain the same number of residues. All other proteins may be similarly constituted. Such views emphasize the need for more work on the isolation and characterization of the partial decomposition products of protein, for much of the evidence hitherto brought forward to support the Bergmann-Niemann hypothesis has been based on inadequate experimental data and has demonstrated nothing more than the hypnotic power of numerology.

\section{TIMBER-SEASONING KILNS}

TWO leaflets have recently been issued by the Forest Research Institute, Dehra Dun, India, on the subject of timber seasoning kilns by M. A. Rehman, Utilization Branch (For. Res. Inst. Public. Vasant Press, Dehra Dun, 1941-42). Leaflet No. 1], "Types of Timber Seasoning Kilns suitable for drying Indian Woods" (1942), describes briefly the various types of seasoning kilns which have been designed or tested by the Forest Institute. It was due to the position in which we found ourselves during the last War in the matter of timber supplies, especially 